\title{
Evidence for a Phenomenological Supersymmetry in Atomic Physics
}

\author{
V. Alan Kostelecký and Michael Martin Nieto \\ Theoretical Division, University of California Los Alamos National Laboratory, Los Alamos, New Mexico 87545
}

(Received 31 July 1984)

\begin{abstract}
We show that supersymmetric quantum mechanics may be used to interrelate the spectra of different atoms and ions. This supersymmetry is broken by electron-electron interactions.
\end{abstract}

PACS numbers: $03.65 . \mathrm{Ge}, 11.30 . \mathrm{Pb}, 31.15 .+\mathrm{q}$

Supersymmetry, which has been used as a theoretical tool in many fields, ${ }^{1}$ has been applied in at least three essentially different ways: as an extension of the Lorentz group of relativistic field theory; as a symmetry relating paired and unpaired nucleons in nuclei; and as a quantum mechanical symmetry. To date, it is only in nuclear physics that experimental evidence has been found to suggest the presence of supersymmetry in nature. ${ }^{2}$ In this Letter we argue that relationships between atomic spectra may be interpreted as being due to a supersymmetry.

The Hamiltonian for supersymmetric quantum mechanics ${ }^{3}$ may be written as $H_{\mathrm{ss}}=H_{+} \oplus H_{-}$, where the bosonic and fermionic components $H_{+}$and $H_{-}$with supersymmetric potential partners $V_{+}$and $V_{-}$are given by

$$
H_{ \pm} \psi_{ \pm \alpha}=\left[-\frac{d^{2}}{d x^{2}}+\frac{1}{4}\left(\frac{d U(x)}{d x}\right)^{2} \mp \frac{1}{2} \frac{d^{2} U(x)}{d x^{2}}\right] \psi_{ \pm \alpha}=\epsilon_{\alpha} \psi_{ \pm \alpha}
$$

The component Hamiltonians have the same spectrum, except that a normalizable ground-state eigenfunction with zero-energy eigenvalue exists only for $H_{+}$:

$$
\psi_{+0}(x) \sim \exp \left[-\frac{1}{2} U(x)\right], \quad \epsilon_{0}=0 .
$$

The radial equation of the hydrogen atom can be written as

$$
\left[-\frac{d^{2}}{d y^{2}}-E_{n}-\frac{1}{y}+\frac{l(l+1)}{y^{2}}\right] \chi_{n l}(y)=0,
$$

where $E_{n}=-1 / 4 n^{2}, y=\left(2 \mu e^{2} / \hbar^{2}\right) r, n \geqslant l+1$, and $\chi_{n l}=R_{n l} y$, with $R_{n l}$ the solution to the usual radial equation. ${ }^{4}$ Note that we have used $y$ rather than the standard variable $\rho=y / n$. Equation (3) may be rewritten in the form (1), with

$$
\begin{aligned}
& V_{+l}=\left(U^{\prime}\right)^{2} / 4-U^{\prime \prime} / 2 \\
&=[2(l+1)]^{-2}-\frac{1}{y}+\frac{l(l+1)}{y^{2}}, \\
& E_{n} \rightarrow \epsilon_{n l}=\left[(l+1)^{-2}-n^{-2}\right] / 4,
\end{aligned}
$$

where the prime denotes differentiation.

Now, we wish to obtain the supersymmetric partner of the hydrogen-atom Hamiltonian. To use Eq. (1), the form of the problem must be truly one-dimensional, i.e., we must select some fixed value of $l$. The solution to Eq. (4a) is then

$$
U(y)=y /(l+1)-2(l+1) \ln y .
$$

Therefore, the supersymmetric partner $V_{-l}$ to the potential $V_{+l}$ is

$$
V_{-l}=[2(l+1)]^{-2}-\frac{1}{y}+\frac{(l+1)(l+2)}{y^{2}} .
$$

Let us first consider the case where $l=0$ ( $s$ orbitals). As mentioned above, the spectrum of $\mathrm{H}_{-l}$ is that of $H_{+l}$ with the ground state removed. Thus, $H_{-0}$ describes a system with the $1 s$ orbitals removed. Physically, this situation is approximated by the $s$ levels of the lithium atom, when two of its three electrons are in the $1 s$ orbitals. In the absence of electron-electron interactions and provided that the valence electron is far enough removed from the core electrons, the effective potential will be Coulombic with a single charge. Therefore, in this approximation, the $s$ levels of the lithium atom may be interpreted as the supersymmetric partner of the hydrogen atom $s$ levels.

We may similarly analyze the case of $l=1$ ( $p$ orbitals). Here, we shall take $H_{+1}$ as modeling the $p$ levels of the boron atom, which has electronic configuration $1 s^{2} 2 s^{2} 2 p^{1}$ in the ground state. We could consider $H_{+1}$ as the hydrogen atom with electronic configurations $n p^{1}$, but the finite lifetime due to spontaneous emission makes this case less natural. In this sense, the most natural supersymmetric partner to the boron atom with configurations $1 s^{2} 2 s^{2} n p^{1}$ is the aluminum atom with configurations $1 s^{2} 2 s^{2} 2 p^{6} 3 s^{2} n p^{1}$. Cases with higher values of 
$l$ may be analyzed similarly.

The Hamiltonians $H_{+l}$ and $H_{-l}$ differ by the quantity $U^{\prime \prime}=2(l+1) / y^{2}$. Physically, this represents the screening potential $\tilde{V}_{l}$. As may be seen from Eqs. (4) and (6), the radial wave functions associated with $H_{+l}$ are related to those associated with $H_{-l}$ by a change in $l$ of one unit. ${ }^{5}$ Mathematically, this is equivalent to a change in the radial angular momentum barrier. However, note that the supersymmetry relates $s$ orbitals to $s$ orbitals, $p$ orbitals to $p$ orbitals, etc.

We can now repeat the process by defining

$$
H_{+l}^{(1)}=H_{-l}+\left[(l+2)^{-2}-(l+1)^{-2}\right] / 4
$$

and finding the corresponding $H_{-l}^{(1)}$. The constant term is added to make the ground-state energy of $H_{+l}^{(1)}$ equal to zero, as is required for supersymmetry. For $l=0$, this is physically equivalent to starting with the $s$-level spectrum of lithium, with the $1 s$ orbitals filled, and determining its supersymmetric partner. We find

$$
\begin{aligned}
& \tilde{V}_{l}^{(1)}=2(l+2) / y^{2}, \\
& V_{-l}^{(1)}=[2(l+2)]^{-2}-\frac{1}{y}+\frac{(l+2)(l+3)}{y^{2}} .
\end{aligned}
$$

In the unbroken-symmetry approximation, the Hamiltonian $H_{-0}^{(1)}$ phenomenologically models the $s$-orbital spectrum of an atom with electronic configurations $1 s^{2} 2 s^{2} 2 p^{6} n s^{1}$, which are those of sodium. Similarly, $H_{-1}^{(1)}$ may be viewed as modeling the $p$-orbital spectrum of gallium.

Repeating this process, we obtain a series of Hamiltonians $H_{+l}^{(k)}$ and $H_{-l}^{(k)}$ which are related to one another via supersymmetry. In general,

$$
\begin{aligned}
& V_{+l}^{(k)}=[2(l+k+1)]^{-2}-\frac{1}{y}+\frac{(l+k)(l+k+1)}{y^{2}} \\
& \tilde{V}_{l}^{(k)}=2(l+k+1) / y^{2}
\end{aligned}
$$

yielding $\quad V_{-l}^{(k)}=V_{+l}^{(k)}+\tilde{V}_{l}^{(k)}$. The Hamiltonians $H_{ \pm l}^{(k)}$ associated with these potentials generate series of spectra that for fixed $l$ are related to one another by supersymmetry. For $l=0$, the natural series interrelates the $s$-orbital levels of the alkali-metal atoms; for $l=1$ it interrelates the $p$-orbital levels of the Group-3 $A$ atoms. Note that, since electronelectron interactions are not taken into account, the spectra of atoms within any given period of the Periodic Table are treated equivalently, e.g., $H_{+1}$ models the spectra of boron through neon while $H_{-1}$ models the spectra of aluminum through argon. We wish to emphasize, however, that we are establishing relationships between real energy levels not connected by physical dipole transitions. The supersymmetry interrelates levels connected by $\Delta n=1$ and $\Delta l=0$, not $\Delta l=1$.

These relationships are broken by electron-electron interactions, in several different ways. First, there is the splitting of levels with the same $n$ but different $l$; this breaks the equivalence of atomic spectra within a fixed period. Second, as a result of the electrons filling the lower states, both the period equivalence and the supersymmetry will be broken when the valence electron occupies lowlying energy levels. Third, the fact that the valence electron wave functions describes an electron cloud distribution which can penetrate the core electron distributions means that the supersymmetry will be broken even for the outer lying levels and will be least apparent for the $s$-state wave functions. To summarize, the supersymmetry should be best for energy levels with large values of $n$ and $l$, when the total number of electrons is small.

Let us now compare these predictions with some experimental values. ${ }^{6}$ Table I lists selected level separations of some alkali-metal atoms. Although the supersymmetry strictly relates only the $s$-orbital levels for atoms in this group, these levels are de-

\begin{tabular}{|c|c|c|c|c|c|c|c|}
\hline \multirow[b]{2}{*}{$n$} & \multirow{2}{*}{$\underset{s / p / d}{\mathrm{H}}$} & \multicolumn{3}{|c|}{$\mathrm{Li}$} & \multicolumn{3}{|c|}{$\mathrm{Na}$} \\
\hline & & $s$ & $p$ & $d$ & $s$ & $p$ & $d$ \\
\hline $8-9$ & 0.0360 & 0.0416 & 0.0366 & $\underline{0.0360}$ & 0.0607 & 0.0496 & $\underline{0.0362}$ \\
\hline $6-7$ & 0.0808 & 0.0980 & 0.0826 & $\overline{0.0810}$ & 0.164 & 0.124 & $\overline{0.0814}$ \\
\hline $4-5$ & 0.245 & 0.329 & 0.255 & 0.247 & 0.746 & 0.477 & 0.249 \\
\hline $3-4$ & 0.533 & 0.781 & 0.555 & $\overline{0.534}$ & 2.57 & 1.33 & $\overline{0.538}$ \\
\hline $2-3$ & 1.52 & 2.72 & 1.60 & . . & . . . & . . & $\overline{\cdot \cdot}$ \\
\hline
\end{tabular}
generate with the $p$ - and $d$-orbital levels in the ab-

TABLE I. Energy differences (in units of $10^{4} \mathrm{~cm}^{-1}$ ) between selected levels of the alkali-metal atoms (Ref. 6). The best agreements with the $\mathrm{H}$ levels are underlined and are those that one would expect from the discussion in the text. 
sence of electron-electron interactions. We, therefore, expect to see the clearest evidence for supersymmetry in the high- $n$ and high- $l$ orbital levels of those atoms with the smallest total number of electrons. This is indeed the case.

A similar comparison may be made for the $l=1$ supersymmetric series of spectra. Unfortunately, relatively few data are available. However, we have indeed found agreements between the spectra of boron and aluminum. This agreement is expected on physical grounds: The spectra of all singly excited atoms are hydrogenic in the limit of large $n$ and $l$ because the valence electron sees a unit effective nuclear charge. Nevertheless, the supersymmetry is interesting in that the atomic spectra associated with $V_{+}^{(k)}$ and $V_{-}^{(k)}$ are described by a single, oneparticle Hamiltonian. In the case of lithium, for example, $H_{-}$provides a simple, phenomenological acceptable approximation to the full many-bodied Hamiltonian. Even the Pauli principle is phenomenologically incorporated in the resulting theory, in the form of the $y^{-2}$ dependence in Eq. (6).

A similar phenomenology may be used to model the spectra of ions. ${ }^{7}$ Let us consider atoms which have been $N$-fold ionized. All the supersymmetric relationships enumerated above occur among the spectra of these ions. Now, however, the energy levels will be separated by amounts which are larger than the neutral-atom case by a factor of $(N+1)^{2}$, because of the increased effective charge. There is still an equivalence between spectra of ions in any given period; both this equivalence and the various supersymmetry series will be broken by the electron-electron interactions, as before. Indeed, experimental values, shown for selected level separations for some Group- $2 A$ ions in Table II, agree with these conclusions.

The informed reader may wonder what the connection is between the supersymmetric method of removing the ground state and the inverse method of Gel'fand and Levitan, ${ }^{8}$ as applied by Abraham and Moses. ${ }^{9}$ While it is true that this second method also removes the ground state, leaving the other eigenvalues unchanged, the potential which must be added to $H+l$ to achieve this is different. We have found the inverse-method screening potential to be given by

$$
\begin{aligned}
& \tilde{V}_{1 l}(y)=2 \phi(l)[\phi(l)-1 /(l+1)+2(l+1) / y], \\
& \phi(l)=y^{(2 l+2)}\left[(2 l+2) ! \sum_{j=0}^{2 l+2} y^{j}(l+1)^{2 l+3-j / j !]^{-1}} .\right.
\end{aligned}
$$

In particular, this is not a supersymmetric result. However, one can verify explicitly that, for $l=0$, this potential gives a solution to the Hamiltonian equation with the ground state removed and that the new, normalized, ground-state wave function $\Lambda(y)$ is that predicted by the inverse method:

$$
\Lambda(y)=\frac{1}{96} e^{-y / 4}\left(y^{4}+6 y^{3}+18 y^{2}+24 y\right)\left(y^{2} / 2+y+1\right)^{-1} .
$$

There does not seem to be a simple physical interpretation of the potential (12). The two methods of removing the ground state are evidently very different on physical grounds. It has been shown ${ }^{10}$ that the supersymmetric $H_{-}$is mathematically related to a special limit of the general inverse method, but this does not seem to play a significant role here.

We should also point out that, although the two phenomenological Hamiltonians have the same discrete spectrum, their eigenfunctions are different. Indeed, in general there can exist an infinity of potentials

TABLE II. Energy differences (in units of $10^{4} \mathrm{~cm}^{-1}$ ) between selected levels of the

\begin{tabular}{|c|c|c|c|c|c|c|c|}
\hline \multirow[b]{2}{*}{$n$} & \multirow{2}{*}{$\begin{array}{l}\text { He II } \\
s / p / d\end{array}$} & \multicolumn{3}{|c|}{ Be II } & \multicolumn{3}{|c|}{$\mathrm{Mg}$ II } \\
\hline & & $s$ & $p$ & $d$ & $s$ & $p$ & $d$ \\
\hline $8-9$ & 0.144 & . . & . . & . . & 0.216 & 0.187 & $\underline{0.146}$ \\
\hline $6-7$ & 0.324 & 0.367 & 0.331 & 0.324 & 0.559 & 0.458 & 0.330 \\
\hline $4-5$ & 0.988 & 1.19 & 1.02 & 0.989 & 2.30 & 1.68 & 1.01 \\
\hline $3-4$ & 2.13 & 2.72 & 2.23 & 2.14 & 6.98 & 4.49 & $\overline{2.18}$ \\
\hline $2-3$ & 6.10 & 8.82 & $\underline{6.46}$ & $\bar{\cdots}$ & . . & . . . & $\bar{\cdots}$ \\
\hline
\end{tabular}
singly ionized Group- $2 A$ atoms (Ref. 6). The best agreements with the He II levels are underlined and are those that one would expect from the discussion in the text. 
whose discrete eigenvalues are identical but whose eigenfunctions are different. ${ }^{8-11}$ Our supersymmetric potential is a special case, but it is an attractive case because the potential has a simple physical interpretation.

We are extremely grateful to Dr. David R. Mariani of Schlumberger-Doll Research for many constructive comments and for a critical reading of the manuscript. We also thank Dr. Michael Grady for several useful discussions and Dr. Kevin Schmidt for his help with a numerical integration checking the analytic result of Eq. (14).

${ }^{1}$ For an overview, see "Supersymmetry in Physics," edited by V. A. Kostelecký and D. K. Campbell (NorthHolland, Amsterdam, to be published).

${ }^{2}$ F. Iachello, Phys. Rev. Lett. 44, 772 (1981), and Nucl. Phys. A370, 284 (1981).

${ }^{3}$ E. Witten, Nucl. Phys. B185, 513 (1981); F. Cooper and B. Freedman, Ann. Phys. (N.Y.) 146, 262 (1983); C. M. Bender, F. Cooper, and B. Freedman, Nucl. Phys.
B219, 61 (1983); E. Gozzi, Phys. Lett. 129B, 432 (1983); M. de Crombrugghe and V. Rittenberg, Ann. Phys. (N.Y.) 151, 99 (1983); M. Bernstein and L. S. Brown, Phys. Rev. Lett. 52, 1933 (1984); R. Akhoury and A. Comtet, to be published.

${ }^{4}$ L. I. Schiff, Quantum Mechanics (McGraw-Hill, New York, 1968).

${ }^{5}$ The predictions of lithium transition probabilities obtained with these unorthodox wave functions are being investigated.

${ }^{6}$ S. Bashkin and J. O. Stoner, Jr., Atomic Energy Levels \& Grotrian Diagrams, Vol. 1: Hydrogen I-Phosphorous XV (Elsevier, New York, 1975).

${ }^{7}$ Evidence for a phenomenological supersymmetry in molecular electronic spectra may be harder to obtain because of the greater role played by electron-electron interactions.

${ }^{8}$ I. M. Gel'fand and B. M. Levitan, Izv. Akad. Nauk SSSR Ser. Mat. 15, 309 (1951) [Am. Math. Soc. Transl. 1, 253 (1955)].

${ }^{9}$ P. B. Abraham and H. E. Moses, Phys. Rev. A 22, 1333 (1980).

${ }^{10} \mathrm{M}$. M. Nieto, to be published.

${ }^{11}$ V. Bargmann, Rev. Mod. Phys. 21, 488 (1949); M. M. Nieto and V. P. Gutschick, Phys. Rev. D 23, 922 (1981); M. M. Nieto, Phys. Rev. D 24, 1030 (1981). 